\title{
Size-dependent oscillator strength and quantum efficiency of CdSe quantum dots controlled via the local density of states
}

\author{
Leistikow, M.D.; Johansen, Jeppe; Kettelarij, A.J.; LodahI, Peter; Vos, W.L.
}

Published in:

Physical Review B Condensed Matter

Link to article, DOI:

10.1103/PhysRevB.79.045301

Publication date:

2009

Document Version

Publisher's PDF, also known as Version of record

Link back to DTU Orbit

Citation (APA):

Leistikow, M. D., Johansen, J., Kettelarij, A. J., Lodahl, P., \& Vos, W. L. (2009). Size-dependent oscillator strength and quantum efficiency of CdSe quantum dots controlled via the local density of states. Physical Review B Condensed Matter, 79(4), 045301. https://doi.org/10.1103/PhysRevB.79.045301

\section{General rights}

Copyright and moral rights for the publications made accessible in the public portal are retained by the authors and/or other copyright owners and it is a condition of accessing publications that users recognise and abide by the legal requirements associated with these rights.

- Users may download and print one copy of any publication from the public portal for the purpose of private study or research.

- You may not further distribute the material or use it for any profit-making activity or commercial gain

- You may freely distribute the URL identifying the publication in the public portal 


\title{
Size-dependent oscillator strength and quantum efficiency of CdSe quantum dots controlled via the local density of states
}

\author{
M. D. Leistikow, ${ }^{1}$ J. Johansen, ${ }^{2}$ A. J. Kettelarij, ${ }^{1}$ P. Lodahl, ${ }^{2}$ and W. L. $\operatorname{Vos}^{1,3, *}$ \\ ${ }^{1}$ Center for Nanophotonics, FOM Institute for Atomic and Molecular Physics (AMOLF), Kruislaan 4071098 SJ, \\ Amsterdam, The Netherlands \\ ${ }^{2}$ DTU Fotonik, Department of Photonics Engineering, Technical University of Denmark, Ørsteds Plads 343, \\ DK-2800 Kgs. Lyngby, Denmark \\ ${ }^{3}$ Complex Photonic Systems (COPS), Faculty of Science and Technology and MESA + Institute for Nanotechnology, \\ University of Twente, 7500 AE Enschede, The Netherlands
}

(Received 21 August 2008; revised manuscript received 25 November 2008; published 5 January 2009)

\begin{abstract}
We study experimentally time-resolved emission of colloidal CdSe quantum dots in an environment with a controlled local density of states (LDOS). The decay rate is measured versus frequency and as a function of distance to a mirror. We observe a linear relation between the decay rate and the LDOS, allowing us to determine the size-dependent quantum efficiency and oscillator strength. We find that the quantum efficiency decreases with increasing emission energy mostly due to an increase in nonradiative decay. We manage to obtain the oscillator strength of the important class of CdSe quantum dots. The oscillator strength varies weakly with frequency in agreement with behavior of quantum dots in the strong confinement limit. Surprisingly, previously calculated tight-binding results differ by a factor of 5 with the measured absolute values. Results from pseudopotential calculations agree well with the measured radiative rates. Our results are relevant for applications of CdSe quantum dots in spontaneous emission control and cavity quantum electrodynamics.

DOI: 10.1103/PhysRevB.79.045301

PACS number(s): 78.47.Cd, 78.67.Hc, 42.50.Ct
\end{abstract}

\section{INTRODUCTION}

Control over spontaneous emission is important for many applications in nanophotonics, such as efficient miniature lasers and light-emitting diodes (LEDs), ${ }^{1,2}$ efficient solar energy collection, ${ }^{3}$ and even biophotonics. ${ }^{4}$ Increasing attention has been given to all solid-state cavity quantum electrodynamics (QED) experiments. ${ }^{5-8}$ For spontaneous emission control the oscillator strength plays a crucial role. The oscillator strength gauges the strength of the interaction of a light source with the light field. The larger the oscillator strength is, the stronger is the interaction between the source and the light field and in cavity QED between source and cavity field.

As light sources in nanophotonics, quantum dots are becoming increasingly popular. Quantum dots are semiconductor nanocrystals with sizes smaller than the exciton Bohr radius. Due to their small size, quantum dots have discrete energy levels. ${ }^{9}$ CdSe colloidal quantum dots in particular have generated enormous interest in recent years because of the tunability of their emission energy over the entire visible range with particle diameter. ${ }^{10}$ Surprisingly no measurements have been done of the emission oscillator strength of these quantum dots, while this is highly important to interpret cavity QED experiments. ${ }^{11}$ The oscillator strength has been investigated only qualitatively using absorption measurements. ${ }^{12-14}$ However, the accuracy of these measurements is limited due to the strong blinking behavior of $\mathrm{CdSe}$ quantum dots, i.e., intermittency in the emission of photons. The oscillator strength determined from absorption is not relevant to emission experiments since the quantum dots in the off state do absorb while they do not contribute to the emission.

In this paper we present quantitative measurements of the oscillator strength and quantum efficiency of colloidal CdSe quantum dots as a function of emission energy and thus dot diameter since the emission energy and diameter are uniquely related. ${ }^{10}$ The oscillator strength of an emitter can be determined by placing it close to an interface. The emission rate is then also affected by emission which is reflected at the interface. This interference leads to a controlled modification of the local density of states (LDOS) allowing us to separate radiative and nonradiative decay rate components. This technique has been pioneered by Drexhage ${ }^{15}$ for dye molecules and used to determine quantum efficiency of $\mathrm{Si}$ nanocrystals, ${ }^{16}$ erbium ions,${ }^{17}$ epitaxially grown InAs quantum dots, ${ }^{18}$ and colloidal CdSe quantum dots. ${ }^{19,20}$ Recently it has been found that the emission oscillator strength can also be determined with this technique. ${ }^{18}$ Here, we place CdSe quantum dots on different distances near a silver interface to quantitatively determine the oscillator strength as a function of emission energy.

\section{EXPERIMENTAL METHODS}

\section{A. Sample fabrication}

The planar samples with controllable LDOS consist of a glass substrate of $24 \times 24 \mathrm{~mm}^{2}$ on which a stack of four different layers is made, as shown in Fig. 1. (1) The first layer is an optically thick $500 \mathrm{~nm}$ layer of silver which is deposited with vapor deposition. (2) Next a layer of $\mathrm{SiO}_{2}$ is evaporated onto the silver. The $\mathrm{SiO}_{2}$ layer has a refractive index of $1.55 \pm 0.01$ at a wavelength of $600 \mathrm{~nm}$ as determined by ellipsometry. The thickness of the $\mathrm{SiO}_{2}$ layer is varied to control the distance $z$ that the quantum dots have to the silver interface. (3) On top of the $\mathrm{SiO}_{2}$ layer, a very thin layer of polymethyl methacrylate (PMMA) that contains the CdSe quantum dots is spin coated. This layer is $\Delta z$ 


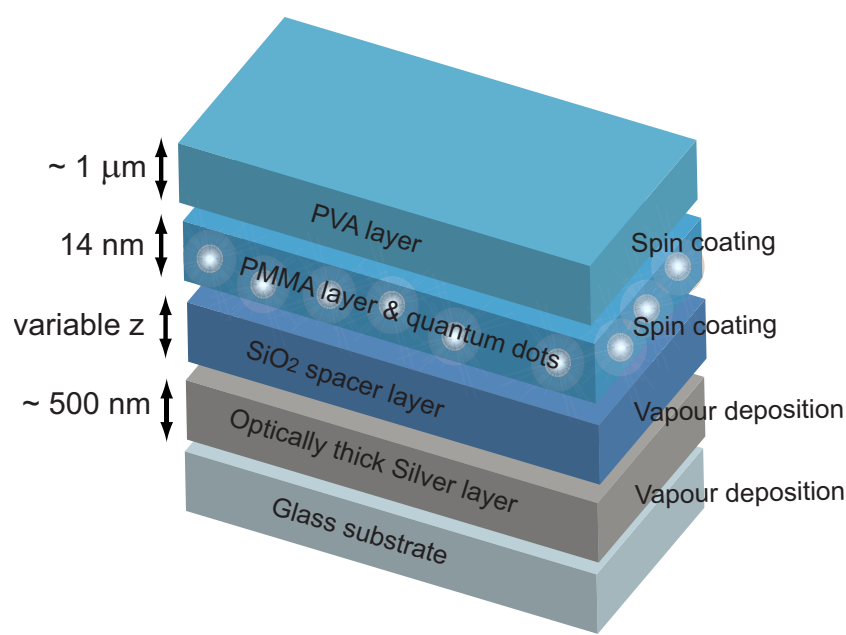

FIG. 1. (Color online) Schematic cross section of the sample used in the measurements. The different layers of the sample are shown together with corresponding thickness and fabrication technique.

$=14 \pm 5 \mathrm{~nm}$ thick determined by profilometry. PMMA has a refractive index of $1.49 \pm 0.01$. (4) On top of the PMMA layer a thick $\sim 1 \mu \mathrm{m}$ layer of polyvinyl alcohol (PVA) is spin coated to avoid reflections from a PMMA/air interface. The PVA is $9.4 \%$ by weight dissolved in a mixture of water and ethanol. Since the PMMA and quantum dots do not dissolve in water and ethanol, the PMMA layer stays intact. PVA has a refractive index of $1.50 \pm 0.01$. All parameters are summarized in Table I.

\section{B. Quantum dots}

CdSe quantum dots with a $\mathrm{ZnS}$ shell are purchased from Evident Technology (Fort Orange, emitting around $600 \mathrm{~nm}$ ). We have performed transmission electron microscopy (TEM) experiments to verify the quantum dot diameter. Figure 2 shows a TEM micrograph of a typical dot that has a diameter of $3.9 \mathrm{~nm}$. From measurements on 98 quantum dots, we have determined the histogram of diameter distributions (see Fig. 3 ). The quantum dots have an average diameter of $D$ $=4.1 \mathrm{~nm}$ with a standard deviation of $0.5 \mathrm{~nm}$. Hence, our quantum dots are smaller than the exciton Bohr radius and therefore the strong confinement for excitons applies to our dots.

The suspension that is spin coated consists of toluene with $0.5 \%$ by weight 495000 molecular weight PMMA and a quantum dot concentration of $1.21 \times 10^{-6} \mathrm{~mol} / \mathrm{liter}$. The

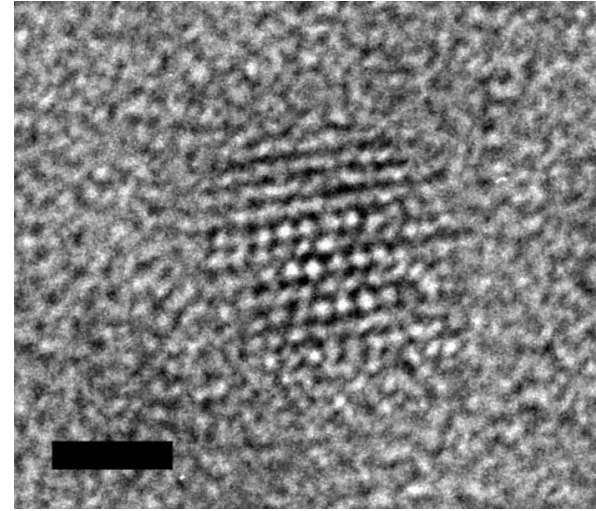

FIG. 2. Transmission electron micrograph of a CdSe quantum dot with a diameter $D=3.9 \mathrm{~nm}$. The fringes from the lattice planes are clearly seen. The scale bar is $2 \mathrm{~nm}$.

quantum dots have an estimated density of $1 / 450 \mathrm{~nm}^{2}$. The quantum dots are thus sufficiently dilute in the PMMA layer to exclude energy-transfer and reabsorption processes between quantum dots. This was verified by measuring that the decay rate was not influenced by laser power or changes in concentration around the used concentration. The sample is contained in a nitrogen purged chamber during measurements to prevent photo-oxidation of the quantum dots.

\section{Optical detection}

The optical setup used in the experiments is schematically shown in Fig. 4. Light from a pulsed frequency doubled $\mathrm{Nd}^{3+}$ :YAG laser (Time Bandwidth Cougar) with an emission wavelength of $532 \mathrm{~nm}$, repetition rate of $8.2 \mathrm{MHz}$, and pulse widths of $11 \mathrm{ps}$ is used. This light is guided into an optical fiber and focused onto the sample by a lens with a focal length of $250 \mathrm{~mm}$, leading to a focus with a diameter of approximately $50 \mu \mathrm{m}$ on the sample.

The light emitted by the quantum dots is collected by a lens, collimated, and focused onto the slit of a prism monochromator (Carl Leiss). The slit width is set to $400 \mu \mathrm{m}$ giving a spectral resolution $\Delta \lambda=6 \mathrm{~nm}$, which is narrow compared to the bandwidth of the LDOS changes. A Hamamatsu multichannel plate photomultiplier tube is used as a photon counter. With this setup it is possible to measure spectra by scanning the monochromator and to measure decay curves of emitters at particular emission frequencies by time-correlated single-photon counting. ${ }^{21}$ This technique measures the time between the arrival of an emitted photon (start) and the laser pulse (stop) with a picosecond resolution. By repeating such

TABLE I. Layer properties.

\begin{tabular}{lccc}
\hline \hline Layer & $\begin{array}{c}\text { Thickness } \\
(\mathrm{nm})\end{array}$ & Refractive index & Fabrication method \\
\hline Silver & 500 & $0.27+4.18 i$ & Vapor deposition \\
$\mathrm{SiO}_{2}$ & Variable $z$ & 1.55 & Vapor deposition \\
$\mathrm{PMMA} \mathrm{CdSe}$ quantum dots & $14 \pm 5$ & 1.49 & Spin coating \\
$\mathrm{PVA}$ & $\sim 1000$ & 1.50 & Spin coating \\
\hline \hline
\end{tabular}




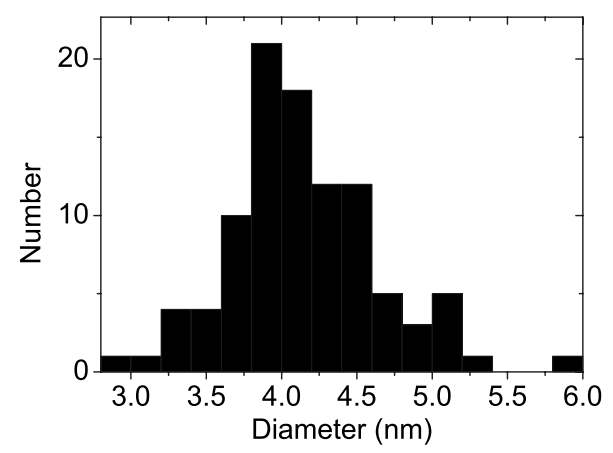

FIG. 3. The distribution in diameter found by analyzing TEM images of 98 quantum dots. The average diameter is $4.1 \mathrm{~nm}$ with a standard deviation of $0.5 \mathrm{~nm}$.

a measurement a histogram of the arrival times is made from which a decay rate can be determined. The time resolution of the setup is 125 ps given by the full width at half maximum of the total instrument response function that is shown in Fig. 6 . The instrument response function is much shorter than the decay curve of CdSe quantum dots, with a typical decay time of $16 \mathrm{~ns}$ in toluene. Therefore, deconvolution of the response function is not necessary to analyze the data.

\section{Data interpretation}

The quantum dots in the polymer layer show a nonexponential decay probably caused by microscopic heterogeneity of the polymer. ${ }^{22}$ Nonexponential behavior has previously been found for CdSe quantum dots in PMMA by Fisher et $a l .{ }^{23}$ even for single-quantum dots. To model the decay curve the data are fitted with a distribution of decay rates as explained in Ref. 24. A function of the following form is used to model the decay curve:

$$
f(t)=\int_{0}^{\infty} \sigma\left(\gamma_{\mathrm{tot}}\right) \exp \left(-\gamma_{\mathrm{tot}} t\right) d \gamma_{\mathrm{tot}}
$$

where the normalized distribution in decay rates is chosen to be lognormal

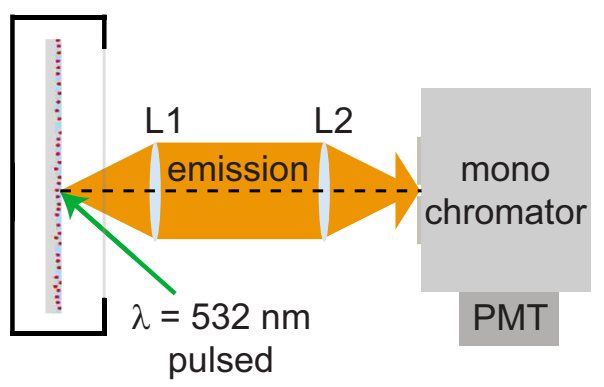

FIG. 4. (Color online) A schematic picture of the experimental setup. Light from the laser excites the quantum dots in a layered sample inside a nitrogen purged chamber. The emitted light is collimated by a lens L1 with $f=12 \mathrm{~cm}$, focused by lens L2 with $f$ $=10 \mathrm{~cm}$ on the entrance slit of a monochromator, and detected by the photomultiplier tube.

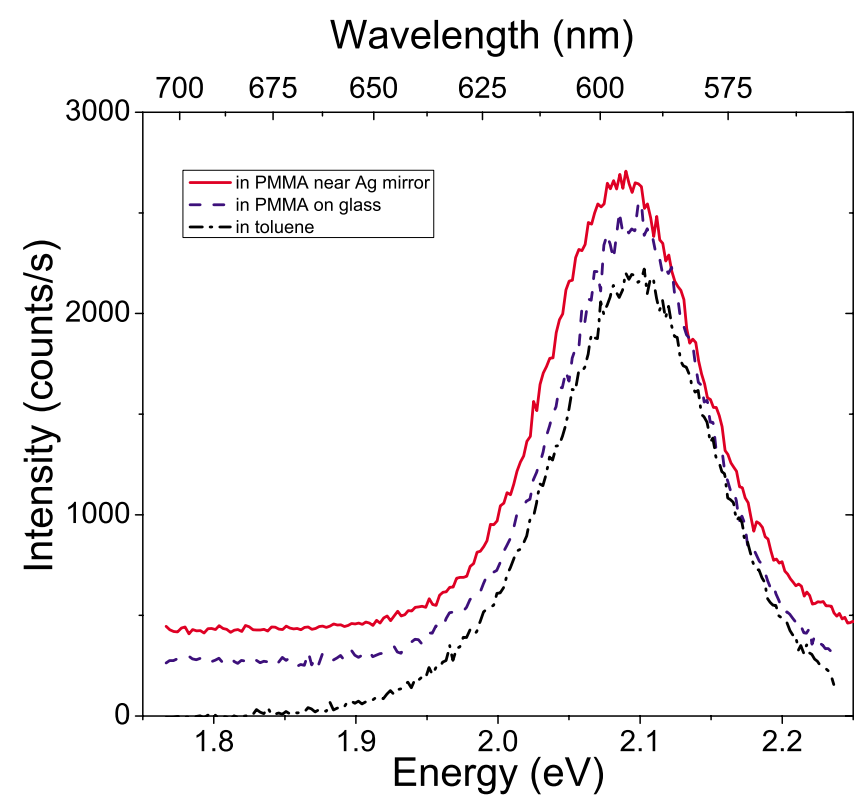

FIG. 5. (Color online) Emission spectra of CdSe quantum dots in toluene suspension, in a planar sample without silver, and in a planar sample with a silver mirror. The spectra are offset for clarity by 200 and 400 counts/s, respectively. The spectrum in PMMA near the mirror and in toluene is scaled to the spectrum in PMMA on glass by a factor of 0.75 .

$$
\sigma(\gamma)=A \exp \left[-\left(\frac{\ln (\gamma)-\ln \left(\gamma_{\mathrm{mf}}\right)}{w}\right)^{2}\right]
$$

The normalization factor $A$ equals $A$ $=\left[\gamma_{\mathrm{mf}} w \sqrt{\pi} \exp \left(w^{2} / 4\right)\right]^{-1}$. The two relevant adjustable parameters that can be extracted from the model are the most frequent decay rate $\gamma_{\mathrm{mf}}$ which is the peak of the lognormal distribution and $\Delta \gamma=2 \gamma_{\mathrm{mf}} \sinh (w)$ which is the $\frac{1}{e}$ width of the lognormal distribution.

Decay rates presented in this paper are an average of decay rates found for at least three measurements performed on different locations on a sample with a particular $\mathrm{SiO}_{2}$ layer thickness. The error in the decay rate is conservatively estimated to be $\pm 3 \%$ which is the maximum difference found between measurements on the same sample.

\section{RESULTS}

\section{A. Experimental results}

In Fig. 5 the emission spectrum of CdSe quantum dots is shown for the quantum dots in toluene, in a planar sample without silver, and in a planar sample with a silver mirror. The peak energies of all three spectra are identical within experimental error. The width of the spectrum is caused by inhomogeneous broadening due to size polydispersity of quantum dots in the ensemble. The homogeneous spectral width of the individual quantum dots is much narrower. ${ }^{25} \mathrm{By}$ selecting a narrow emission energy window, quantum dots of a particular diameter are selected. Within experimental error there is no difference between the widths of the emission spectra in the different environments, indicating that there is 


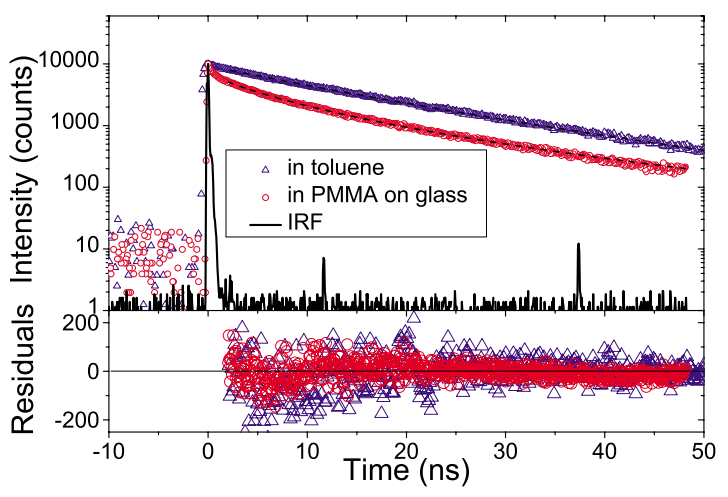

FIG. 6. (Color online) Decay curves of quantum dots at the emission peak at $2.08 \mathrm{eV}$ in PMMA on glass with a top layer of PVA (red circles) and these quantum dots in toluene suspension (blue triangles). The instrument response function (IRF) is indicated by the black line. The peaks in the IRF near 12 and 36 ns are related to the pulse picker of the laser. The decay curves are fitted with a lognormal distribution of decay rates. Residuals are shown in the bottom panel.

no spectral broadening due to the polymer environment.

In Fig. 6 decay curves are shown at the emission peak at $2.08 \mathrm{eV}$ for an ensemble of quantum dots in toluene suspension and in a planar layer without mirror. The quantum dots in toluene show a single exponential decay as expected, giving a decay rate $\gamma=0.061 \pm 0.002 \mathrm{~ns}^{-1}$. Fitting the data with a single exponential gives a value of 1.94 for the goodness of fit $\chi_{\text {red }}^{2}$ indicative of a reasonable fit. ${ }^{26}$

The lognormal distribution of decay rates can be fitted to the decay curve of quantum dots inside PMMA and appears to be a good fit with $\chi_{\text {red }}^{2}=1.49$. For the quantum dots inside the PMMA layer, $\gamma_{\mathrm{mf}}=0.084 \pm 0.002 \mathrm{~ns}^{-1}$. This decay rate indicates the peak in the distribution. The decay of spontaneous emission from quantum dots in toluene suspension can also be fitted with a lognormal distribution of decay rates, giving $\chi_{\text {red }}^{2}=1.71$. The distribution of decay rates in toluene is characterized by $\gamma_{\mathrm{mf}}=0.063 \pm 0.002 \mathrm{~ns}^{-1}$ close to the value for the decay rate $\gamma=0.061 \pm 0.002 \mathrm{~ns}^{-1}$ found from a single exponential decay. In Fig. 7 the lognormal distributions of decay rates are shown for the decay curve of quantum dots in toluene and in the polymer layer. The distribution of decay rates for quantum dots in polymer is much broader than the distribution found for quantum dots in toluene. When a curve is modeled with a single exponential decay, the decay rate distribution reduces to a delta function (indicated in black). The decay rate at the peak of the distribution, the most frequent decay rate, characterizes the decay in the measurement best as supported by the fact that the single exponential rates $\gamma$ and $\gamma_{\mathrm{mf}}$ for decay in toluene are equal within experimental error. The most frequent decay rate will be used in our further analysis.

Measurements of decay rates for two planar samples with different $\mathrm{SiO}_{2}$ layer thicknesses $(z=73 \mathrm{~nm}$ and $z=166 \mathrm{~nm}$, respectively, for samples 1 and 2) are shown in Fig. 8 for quantum dots that emit at the peak emission energy of 2.08 $\mathrm{eV}$. Nonexponential and significantly different decay curves are found for quantum dots that have different distances to the silver interface. The quantum dots in sample 1 clearly

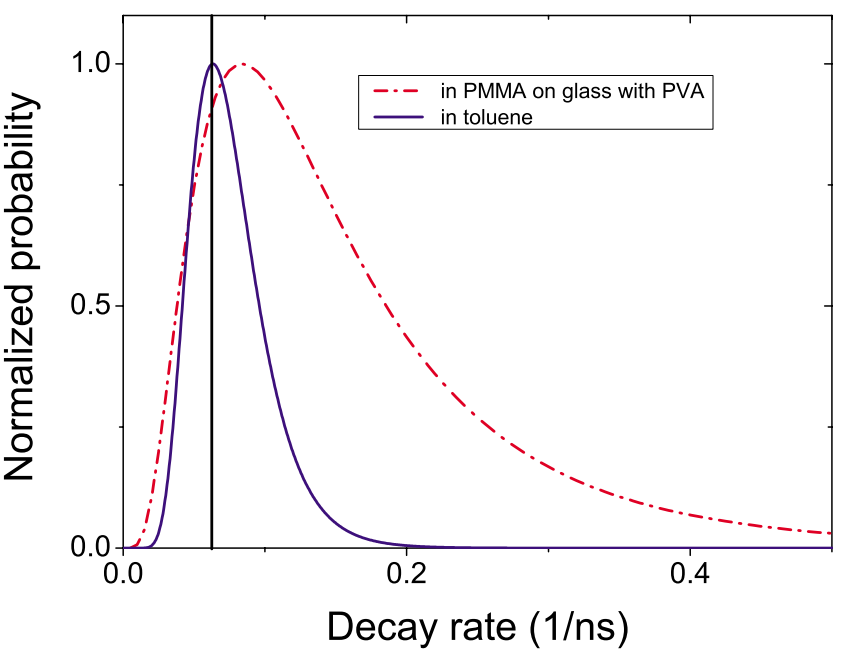

FIG. 7. (Color online) Lognormal distribution of decay rates of quantum dots in a PMMA layer on glass with a PVA cover layer and for quantum dots in toluene resulting from fits in Fig. 6. The black line shows the delta function distribution for single exponential fit.

decay faster than those in sample 2. The experimental curves are fitted with a lognormal distribution of decay rates. The residuals shown in the bottom panel are randomly distributed around a mean value of zero, signaling a good fit. Indeed $\chi_{\text {red }}^{2}$ is 0.72 and 1.44 for samples 1 and 2, respectively, close to the ideal value of 1 , confirming that the decay curves are well modeled by a lognormal distribution of decay rates.

\section{B. Model of decay rates}

Results for the most frequent decay rate for different distances to the interface are presented in Fig. 9 for two different emission energies. The most frequent decay rate decreases with increasing distance to the silver mirror. The measured decay rate $\gamma_{\text {tot }}$ is the sum of radiative $\gamma_{\text {rad }}$ and nonradiative $\gamma_{\text {nrad }}$ decay rates, $\gamma_{\text {tot }}=\gamma_{\text {rad }}+\gamma_{\text {nrad }}$. From Fermi's golden rule the radiative decay rate is proportional to the projected LDOS $\rho(\omega, z)$. Therefore, the total decay rate can be expressed as

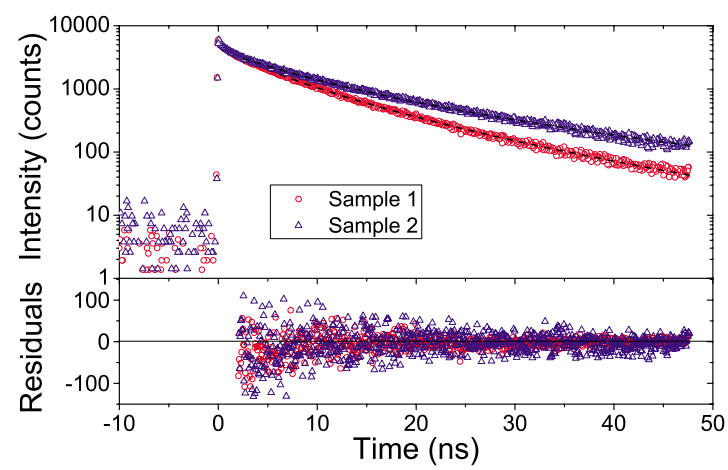

FIG. 8. (Color online) Decay curves for quantum dot samples with different $\mathrm{SiO}_{2}$ layer thicknesses $z=73 \mathrm{~nm}$ and $z=166 \mathrm{~nm}$, respectively, for samples 1 and 2 measured at an emission energy of $2.08 \mathrm{eV}$. The decay curves are fitted with a lognormal distribution of decay rates. Residuals are shown in the bottom panel. 


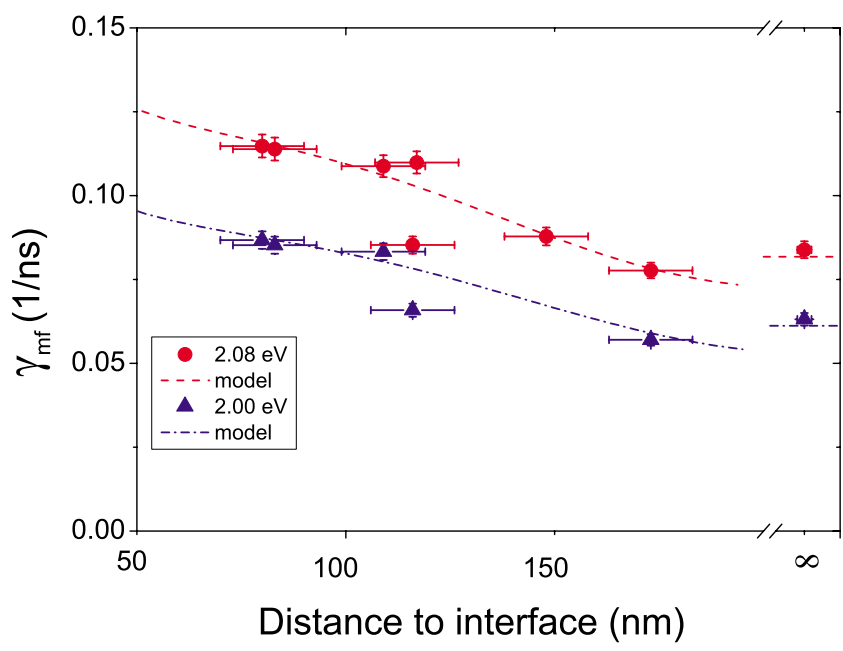

FIG. 9. (Color online) Most frequent decay rate $\gamma_{\mathrm{mf}}$ versus distance to the interface for an emission energy of $2.08 \mathrm{eV}$ (circles) and $2.00 \mathrm{eV}$ (triangles). The lines show calculations of the decay rate using the model developed by Chance et al. (Ref. 27).

$$
\gamma_{\mathrm{tot}}(\omega, z)=\gamma_{\mathrm{nrad}}(\omega)+\gamma_{\mathrm{rad}}^{\mathrm{hom}}(\omega) \frac{\rho(\omega, z)}{\rho_{\mathrm{hom}}(\omega)} .
$$

Here, $\rho_{\text {hom }}(\omega)$ is the LDOS in a homogeneous medium. The LDOS near an interface has been calculated using a theory developed by Chance et al. ${ }^{27}$ As a model an interface between two semi-infinite media has been used, with $n_{1}$ $=0.27+4.18 i(\text { Ag layer })^{28}$ and $n_{2}=1.52(\mathrm{SiO} 2, \mathrm{PMMA}$, and PVA). The LDOS is calculated for dipoles oriented parallel or perpendicular to the interface. Our measurements are performed on an ensemble of quantum dots that are randomly oriented with respect to the interface. This situation differs from self-assembled dots that are strongly oriented. ${ }^{18} \mathrm{~A}$ decay measurement $f(t)$ for an ensemble of emitters can be described by the following expression: ${ }^{29,30}$

$$
f(t)=\frac{I_{0}}{2 \pi} \int_{0}^{2 \pi} d \phi \int_{0}^{\pi / 2} d \theta A(\theta, \phi) \gamma(\theta, \phi) e^{-\gamma(\theta, \phi) t} \sin \theta .
$$

The term $A(\theta, \phi)$ accounts for angle dependence of absorption, emission, and detection. CdSe quantum dots do not have angle-dependent absorption. ${ }^{31}$ Moreover, CdSe quantum dots are known to have a two-dimensional (2D) transition dipole described by a "dark axis" along the $c$ axis of the nanocrystal and a "bright plane" perpendicular to this axis in which the transition dipole can be oriented. ${ }^{31,32}$ Since the quantum dots have a $2 \mathrm{D}$ dipole, the emission is less directional than if it were a one-dimensional (1D) dipole. Because the angle dependence of the emission and detection plays a small role, the factor $A(\theta, \phi)$ can be safely taken to be independent of $\theta$ and $\phi$. Near an interface, the decay rate $\gamma$ is no longer dependent on $\phi$ and is given by $\gamma(\theta)=\gamma_{\|} \cos ^{2} \theta$ $+\frac{\left(\gamma_{\|}+\gamma_{\perp}\right)}{2} \sin ^{2} \theta$, where $\theta$ is the angle between the dark axis of the quantum dot and the normal to the interface as defined in Fig. 10. Therefore, carrying out the integral over $\phi$ results in

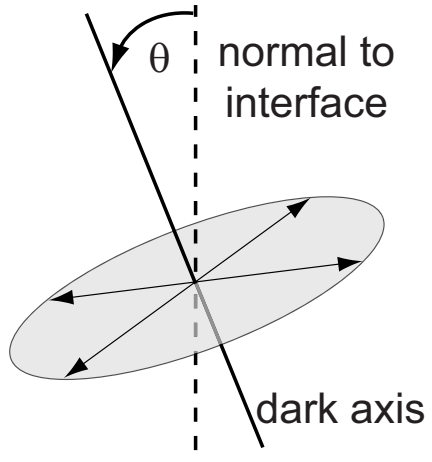

FIG. 10. The angle $\theta$ is the angle between the dark axis of the CdSe quantum dot and the normal to the interface.

$$
\begin{aligned}
f(t)= & I_{0} \int_{0}^{\pi / 2}\left(\gamma_{\|} \cos ^{2} \theta+\frac{\left(\gamma_{\|}+\gamma_{\perp}\right)}{2} \sin ^{2} \theta\right) \\
& \times e^{-\left(\gamma_{\|} \cos ^{2} \theta+\left(\gamma_{\|}+\gamma_{\perp}\right) / 2 \sin ^{2} \theta\right) t} \sin \theta d \theta .
\end{aligned}
$$

If $\gamma_{\|}=\gamma_{\perp}$ the decay curve shows a single exponential decay. When $\gamma_{\|}$and $\gamma_{\perp}$ have different values a multiexponential decay is found. In our experiment, $\gamma_{\|}$and $\gamma_{\perp}$ only differ by about at most $10 \%$. If $f(t)$ is calculated for an intensity range of 3 decades relevant to our experiment, a single exponential decay is found to a very high precision with a decay rate given by $\gamma_{\text {tot }}=\frac{1}{3} \gamma_{\perp}+\frac{2}{3} \gamma_{\|}$. This isotropic decay rate is also used for experiments with atoms near an interface, where the atom has a rotating transition dipole moment. ${ }^{33}$

\section{Discussion}

The lines in Fig. 9 show the calculated isotropic decay rate versus distance to the interface. The calculations are in very good agreement with the data.

By calculating the LDOS for each distance, the distance axis in Fig. 9 can be converted to an LDOS axis. In Fig. 11 the results are shown for two different emission frequencies together with a linear fit. Very good agreement between experiments and theory is observed as expected from Fermi's golden rule. For an emission energy of $2.08 \mathrm{eV}, \gamma_{\mathrm{nrad}}$

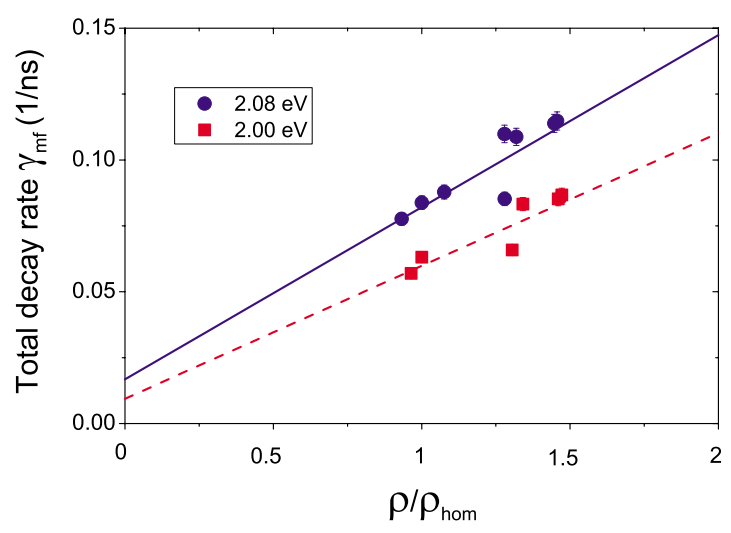

FIG. 11. (Color online) The decay rate versus the normalized isotropic LDOS for two different emission energies. Data are fitted with a linear function as expected from Fermi's golden rule. 


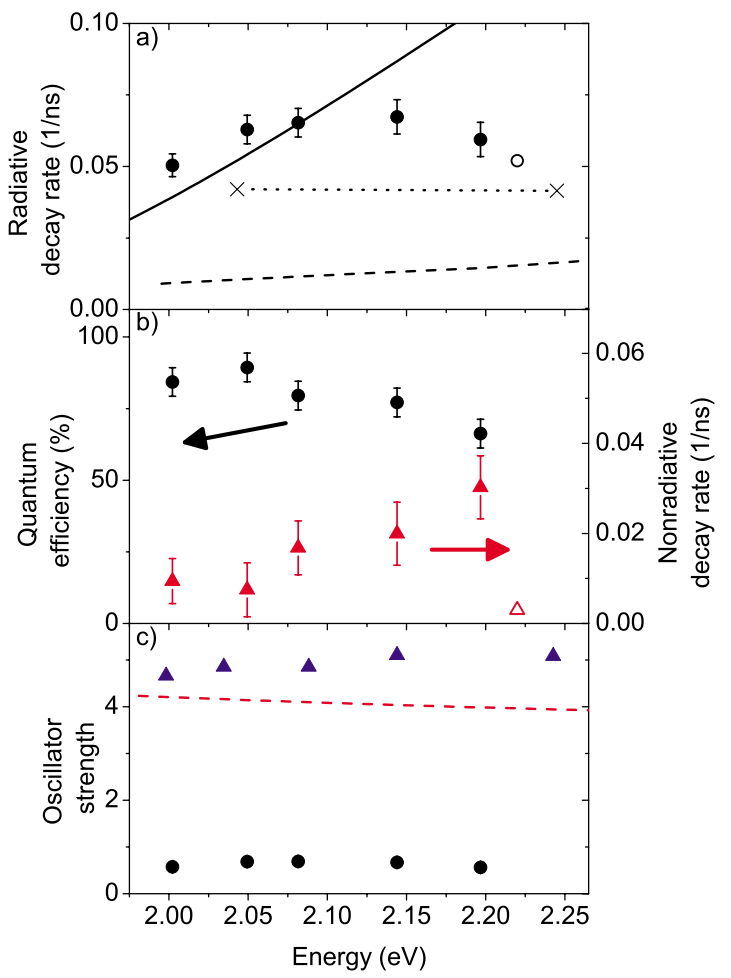

FIG. 12. (Color online) (a) Radiative decay rate (filled circles) determined from the linear fit in Fig. 11 shown versus emission energies. One data point by Brokmann et al. (Ref. 19) is plotted with the open circle. A model for a multilevel exciton from Ref. 34 is shown with a solid line. The dashed line is a tight-binding calculation of radiative decay rate (Ref. 35). The crosses connected with the dotted line are the results from pseudopotential calculations (Ref. 36). (b) Quantum efficiency (circles) and nonradiative decay rate (filled triangles) versus emission energies. The open triangle is the result for nonradiative decay from Ref. 19. (c) Oscillator strength for different emission energies (circles) together with a model describing a strongly confined quantum dot [Eq. (7), dashed line] and results from tight-binding calculations (triangles) (Ref. 38).

$=0.017 \pm 0.006 \mathrm{~ns}^{-1}$ and $\gamma_{\mathrm{rad}}^{\mathrm{hom}}=0.065 \pm 0.005 \mathrm{~ns}^{-1}$, giving a quantum efficiency of $(80 \pm 5) \%$.

In Fig. 12(a) the homogeneous radiative decay rate $\gamma_{\mathrm{rad}}^{\text {hom }}$ is shown as a function of the emission energy. The homogeneous radiative decay rate is observed to first increase and then decrease with emission frequency. The radiative decay rate found by Brokmann et al. ${ }^{19}$ corresponds very well to our data. It should be noted that we derive the homogeneous radiative decay from the most frequent decay rate of the distribution. Since our data agree very well with the decay rate found using a single exponential model and a much shorter integration time, ${ }^{19}$ this corroborates our choice for the most frequent decay rate as the parameter that describes the decay curves best. Our results also validate the choice for the isotropic decay rate model assumed by Brokmann et al.

Previously van Driel et al. ${ }^{34}$ reported that the total decay rate (which is the sum of radiative and nonradiative decay rates) of CdSe colloidal quantum dots increases with emission energy in agreement with our measurements. A theory was developed for the radiative decay rate as a function of frequency. For an ideal two-level exciton, the radiative decay rate should be proportional to frequency. If a multilevel model of the exciton is considered this increase will be supralinear. In Ref. 34 the model for the excitonic multilevel emitter shows agreement with the total decay rate data for CdSe quantum dots and excellent agreement for CdTe dots. The assumption was also made that the total decay rate is equal to the radiative decay rate. However, this is not valid, as can be seen in Fig. 12(a). Results for the multilevel exciton model for radiative decay rate are plotted in Fig. 12(a). The model does not match the data, indicating that the multilevel exciton model is not a correct description for $\mathrm{CdSe}$ quantum dots. The results of a tight-binding calculation ${ }^{35}$ have values $75 \%$ lower than in the experiment, which thus also do not describe CdSe quantum dots. Califano et al. ${ }^{36}$ calculated the room-temperature radiative decay rate via a pseudopotential calculation. Good agreement between this calculation and our data is seen, both qualitatively and quantitatively.

The quantum efficiency for different emission energies is shown in Fig. 12(b). The quantum efficiency is found to be between $66 \%$ and $89 \%$ depending on emission energy. These values are significantly higher then the value stated by the supplier Evident, (30-50)\%. This latter value was determined by comparing the emission intensity to an emitter with known quantum efficiency. ${ }^{26}$ This method leads to an underestimation of the quantum efficiency because it depends on absorption of light. CdSe quantum dots show strong blinking behavior, and quantum dots that are in the off state do absorb light but do not emit. These quantum dots are probed with an absorption measurement, while there is no contribution to the emission. This causes an underestimation of the quantum efficiency in absorption measurements.

On the right axis in Fig. 12(b) the nonradiative decay rate is plotted. The nonradiative decay rate increases with emission energy or equivalently decreases with quantum dot size. This is probably due to the fact that for smaller quantum dots the surface is relatively more important. Since the surface is a source of nonradiative decay, this decay rate is increased for smaller quantum dots. An increased nonradiative decay rate for smaller quantum dots agrees with previous results for CdSe quantum dots ${ }^{37}$ as well as for epitaxially grown InAs quantum dots. ${ }^{18}$ The nonradiative decay rate found by Brokmann et al. ${ }^{19}$ for a different batch of quantum dots is lower than our results. The difference could very well be caused by a difference in the $\mathrm{ZnS}$ capping layer since this drastically changes the nonradiative decay.

The emission oscillator strength $f_{\text {osc }}$ of the transition can be calculated from the homogeneous radiative decay rate via $^{39}$

$$
f_{\text {osc }}(\omega)=\frac{6 m_{e} \epsilon_{0} \pi c^{3}}{q^{2} n \omega^{2}} \gamma_{\mathrm{rad}}^{\mathrm{hom}}(\omega),
$$

where $m_{e}$ is the electron mass, $\epsilon_{0}$ is the vacuum permittivity, $c$ is the speed of light, $q$ is the electron charge, and $n$ is the refractive index of the surrounding material. For an emission energy of $2.08 \mathrm{eV}, f_{\text {osc }}=0.69 \pm 0.04$. We have therefore derived the oscillator strength of colloidal quantum dots by measuring the photoluminescent emission from these quan- 


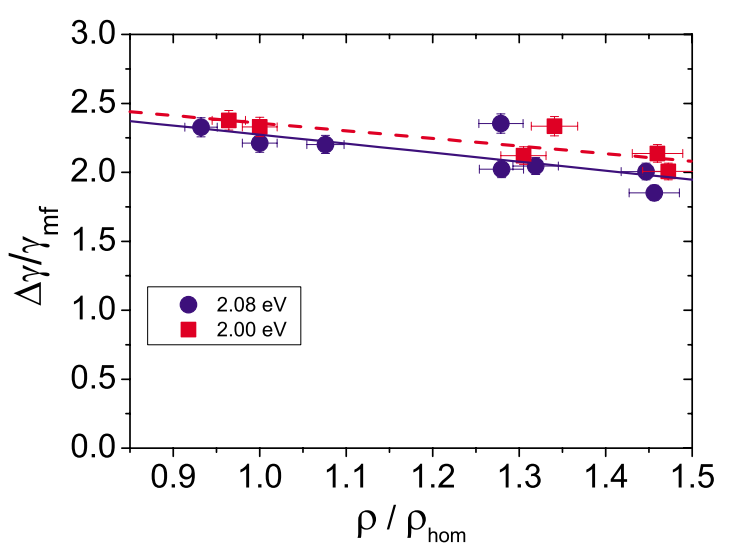

FIG. 13. (Color online) Relative width of the lognormal distribution versus LDOS for emission energies of 2.08 and $2.00 \mathrm{eV}$. The lines are linear fits of the data.

tum dots. Previous qualitative experiments to determine the relation between oscillator strength and size of quantum dots used the absorption spectrum of the quantum dots. ${ }^{12-14}$ The absorption oscillator strength is not necessarily equal to the emission oscillator strength since our measurement is only sensitive to quantum dots that emit light and are in the on state, while absorption measurements probe all quantum dots of the strongly blinking ensemble, including dots that are in the off state.

In Fig. 12(c) the experimentally found oscillator strength is shown for different emission energies. The oscillator strength is only weakly dependent on energy: at first showing a slight increase which is followed by a slight decrease with increasing emission energy. Indeed for quantum dots in the strong confinement regime the oscillator strength is expected to be only weakly dependent on emission energy since, in this regime, the wave functions of electron and hole overlap completely independent of quantum dot size. ${ }^{9,40}$ To verify whether this overlap between electron and hole is indeed unity, the wave functions for electron and hole were calculated using a finite-element method for a simple effectivemass quantum dot model. The overlap was calculated for a spherical CdSe quantum dot core with a $2 \mathrm{~nm} \mathrm{ZnS}$ shell. As expected, the overlap deviated from unity by only $10^{-4}$ for core radii ranging from 2 to $4 \mathrm{~nm}$.

In the strong confinement limit the oscillator strength is given by 40

$$
f_{\text {osc }}=\frac{3}{4} \frac{a_{B}^{* 3}}{R^{3}} \frac{\omega_{\text {bulk }}}{\omega_{\text {dot }}} f_{\text {bulk }} \frac{\frac{4}{3} \pi R^{3}}{\frac{1}{2} \frac{\sqrt{3}}{2} a^{2} c}=\frac{4}{\sqrt{3}} \pi \frac{a_{B}^{* 3}}{a^{2} c} \frac{\omega_{\text {bulk }}}{\omega_{\text {dot }}} f_{\text {bulk }},
$$

where $f_{\text {osc }}$ is the oscillator strength of the quantum dot, $f_{\text {bulk }}$ is the oscillator strength in bulk per chemical CdSe unit, $a_{B}^{*}$ is the exciton Bohr radius, $R$ is the radius of the quantum dot, $\omega_{\text {bulk }}$ is the bulk emission frequency, $\omega_{\text {dot }}$ is the emission frequency of the quantum dot, and $a$ and $c$ are the hexagonal lattice constants of $\mathrm{CdSe}$ (wurtzite structure). For $a_{B}^{*}$ $=5.4 \mathrm{~nm}, a=0.4302 \mathrm{~nm}, c=0.7014 \mathrm{~nm}, f_{\text {bulk }}=5 \times 10^{-4}$ per chemical CdSe unit, ${ }^{13}$ and $\omega_{\text {bulk }}=2.79 \times 10^{15} \mathrm{rad} / \mathrm{s}$ the ex- pected curve is shown in Fig. 12(c). The calculated values are a factor of 5 larger than the experimentally found values. The oscillator strength has also been calculated by Ramaniah and Nair ${ }^{38}$ by a tight-binding approach and was found to be 4.9 for spherical CdSe quantum dots with a radius of 2.07 $\mathrm{nm}$. Interestingly, if the oscillator strength is calculated from the radiative decay rate found by the tight-binding approach $^{35}$ from Fig. 12(a) oscillator strengths, a factor of 4 lower than our experimental values are found, which is opposite to Ref. 38. The cause of the differences between strong confinement [Eq. (7)], tight-binding calculations and our data is not known. However, qualitatively in all cases a weak dependence on emission energy is found that slightly decreases for higher emission energy, in agreement with our results.

Results from absorption measurements ${ }^{12,13}$ also find that the oscillator strength is independent of radius. Leatherdale et $a .^{14}$ found a different behavior, seeing a linear relation between oscillator strength per volume and radius instead of a cubic dependence.

Remarkably, for such a widely studied quantum dot as $\mathrm{CdSe}$, theoretical understanding of the emission behavior is limited. Only the pseudopotential calculations show quantitative agreement with our experiments while tight-binding results show either underestimation or overestimation. Therefore, a fundamental study is warranted to interpret the large variations among the theoretical predictions.

\section{CONCLUSION}

In conclusion, we have separately determined the radiative and nonradiative decay of CdSe quantum dots by modifying the LDOS in a controlled way and measuring the total decay rate. This allows us to quantitatively determine the oscillator strength and quantum efficiency versus emission frequency. The nonradiative decay rate increases with emission energy corresponding to a decrease in quantum efficiency. The radiative decay rate first increases and then decreases with energy. This leads to the conclusion that the increase in total decay rate with energy measured previously is due to an increasing nonradiative component. The emission oscillator strength as a function of emission energy is determined with unprecedented accuracy since this quantity is determined directly from emission experiments. The oscillator strength is weakly size dependent, which is expected in the strong confinement regime. The oscillator strength is found to be on the order of 0.7. Previous calculations of the radiative decay rate by pseudopotential method agree well with our results, whereas the results from tight binding differ considerably. The limited oscillator strength makes the CdSe colloidal quantum dots less suited for cavity QED experiments. On the other hand, the quantitative determination of the oscillator strength paves the way for an $a b$ initio understanding of spontaneous emission control. ${ }^{7}$

\section{ACKNOWLEDGMENTS}

We thank Oscar Bok, Hans Zeijlemaker, and Chris Rétif for the help with sample preparation and Pedro de Vries, Ad 
Lagendijk, and Floris van Driel for helpful discussions. This work was supported by the Stichting Fundamenteel Onderzoek der Materie (FOM) that is financially supported by the Nederlandse Organisatie voor Wetenschappelijk Onderzoek (NWO) and NWO VICI.

\section{APPENDIX: CONCLUSIONS FOR RELATIVE WIDTH OF THE DISTRIBUTION}

In this work results of the effect of modified LDOS on the most frequent decay rate are presented. This most frequent decay rate is found by fitting a lognormal distribution of decay rates to the experimental decay curves. The other independent fitting parameter in this fit is the relative width of the lognormal distribution. In this appendix results for the relative width are presented.

In Fig. 13 the relative width, defined as $\frac{\Delta \gamma}{\gamma_{\mathrm{mf}}}$, is plotted versus normalized local density of states for emission energies of 2.08 and $2.00 \mathrm{eV}$. For increasing LDOS the relative width decreases linearly. Increasing the LDOS effectively increases the quantum efficiency because the radiative decay rate is increased while the nonradiative decay rate is constant. For increasing quantum efficiency the distribution in decay rates gets narrower, giving a strong indication that the width of the distribution is determined by the nonradiative decay rate confirming the proposition by Fisher et al. ${ }^{23}$

In Fig. 14 the relative width measured in the homogeneous environment with $\mathrm{LDOS}=1$ is plotted versus the extracted quantum efficiency for each emission energy. The

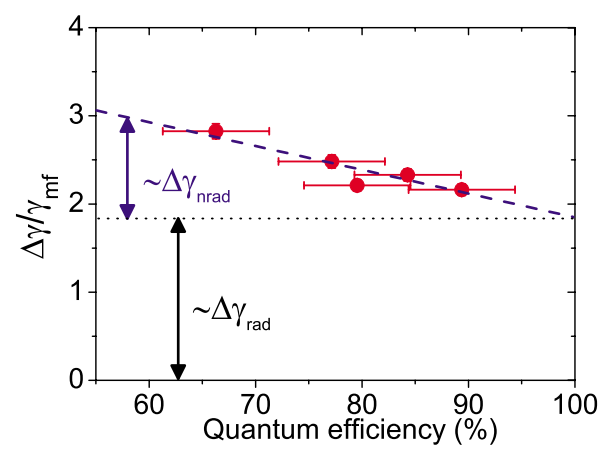

FIG. 14. (Color online) Measurements of relative width for a homogeneous system $(\mathrm{LDOS}=1)$ plotted versus the extracted quantum efficiency [see Fig. 12(b)] together with a linear fit.

same trend is found. For increasing quantum efficiency the relative width of the distribution decreases linearly. When the quantum efficiency is $100 \%$, the decay rate is purely radiative. If the width in the distribution of decay rates is only caused by the nonradiative rate, the width should be zero at $100 \%$ efficiency. This is not the case, indicating that there is a distribution in radiative decay rate as well. Vallée et $a l .^{22}$ also found distributions of decay rates for single dye in polymer and attributed this to local-density variations in the surrounding polymer matrix causing a distribution in radiative decay rate. In conclusion, our data show that there is both a distribution in nonradiative and radiative decay rate that cause the distribution in total decay rate.

*www.photonicbandgaps.com

${ }^{1}$ E. Yablonovitch, Phys. Rev. Lett. 58, 2059 (1987).

${ }^{2}$ H.-G. Park, S.-H. Kim, S.-H. Kwon, Y.-G. Ju, J.-K. Yang, J.-H. Baek, S.-B. Kim, and Y.-H. Lee, Science 305, 1444 (2004).

${ }^{3}$ M. Grätzel, Nature (London) 414, 338 (2001).

${ }^{4}$ C. Blum, A. P. Mosk, I. S. Nikolaev, V. Subramaniam, and W. L. Vos, Small 4, 492 (2008).

${ }^{5}$ T. Yoshie, A. Scherer, J. Hendrickson, G. Khitrova, H. M. Gibbs, G. Rupper, C. Ell, O. B. Schekin, and D. G. Deppe, Nature (London) 432, 200 (2004).

${ }^{6}$ J. P. Reithmaier, G. Sek, A. Loffler, C. Hofmann, S. Kuhn, S. Reitzenstein, L. V. Keldysh, V. D. Kulakovskii, T. L. Reinecke, and A. Forchel, Nature (London) 432, 197 (2004).

${ }^{7}$ P. Lodahl, A. F. van Driel, I. S. Nikolaev, A. Irman, K. Overgaag, D. Vanmaekelbergh, and W. L. Vos, Nature (London) 430, 654 (2004)

${ }^{8}$ E. Peter, P. Senellart, D. Martrou, A. Lemaître, J. Hours, J. M. Gérard, and J. Bloch, Phys. Rev. Lett. 95, 067401 (2005).

${ }^{9}$ L. E. Brus, J. Chem. Phys. 80, 4403 (1984).

${ }^{10}$ A. L. Efros, M. Rosen, M. Kuno, M. Nirmal, D. J. Norris, and M. Bawendi, Phys. Rev. B 54, 4843 (1996).

${ }^{11}$ N. Le Thomas, U. Woggon, O. Schöps, M. V. Artemyev, M. Kazes, and U. Banin, Nano Lett. 6, 557 (2006).

${ }^{12}$ O. Schmelz, A. Mews, T. Basche, A. Herrmann, and K. Müllen, Langmuir 17, 2861 (2001).

${ }^{13}$ A. Striolo, J. Ward, J. M. Prausnitz, W. J. Parak, D. Zanchet, D.

Gerion, D. Milliron, and A. P. Alivisatos, J. Phys. Chem. B 106, 5500 (2002).

${ }^{14}$ C. A. Leatherdale, W. K. Woo, F. V. Mikulec, and M. G. Bawendi, J. Phys. Chem. B 106, 7619 (2002).

${ }^{15}$ K. H. Drexhage, J. Lumin. 1-2, 693 (1970).

${ }^{16}$ R. J. Walters, J. Kalkman, A. Polman, H. A. Atwater, and M. J. A. de Dood, Phys. Rev. B 73, 132302 (2006).

${ }^{17}$ E. Snoeks, A. Lagendijk, and A. Polman, Phys. Rev. Lett. 74, 2459 (1995).

${ }^{18}$ J. Johansen, S. Stobbe, I. S. Nikolaev, T. Lund-Hansen, P. T. Kristensen, J. M. Hvam, W. L. Vos, and P. Lodahl, Phys. Rev. B 77, 073303 (2008).

${ }^{19}$ X. Brokmann, L. Coolen, M. Dahan, and J. P. Hermier, Phys. Rev. Lett. 93, 107403 (2004).

${ }^{20}$ J. Y. Zhang, X. Y. Wang, and M. Xiao, Opt. Lett. 27, 1253 (2002).

${ }^{21}$ D. V. O'Connor and D. Philips, Time Correlated Single Photon Counting (Academic, London, 1984).

${ }^{22}$ R. A. L. Vallée, N. Tomczak, L. Kuipers, G. J. Vancso, and N. F. van Hulst, Phys. Rev. Lett. 91, 038301 (2003).

${ }^{23}$ B. R. Fisher, H. Eisler, N. E. Stott, and M. G. Bawendi, J. Phys. Chem. B 108, 143 (2004).

${ }^{24}$ A. F. van Driel, I. S. Nikolaev, P. Vergeer, P. Lodahl, D. Vanmaekelbergh, and W. L. Vos, Phys. Rev. B 75, 035329 (2007).

${ }^{25}$ S. A. Empedocles, D. J. Norris, and M. G. Bawendi, Phys. Rev. Lett. 77, 3873 (1996). 
${ }^{26}$ J. R. Lakowicz, Principles of Fluorescence Spectroscopy (Kluwer Academic, New York, 1999).

${ }^{27}$ R. R. Chance, A. Prock, and R. Silbey, Advances in Chemical Physics (Wiley, New York, 1978), Vol. 37.

${ }^{28}$ D. R. Lide, CRC Handbook of Chemistry and Physics, 88th ed. (CRC, Boca Raton, 2007), pp. 2007-2008.

${ }^{29}$ N. Danz, J. Heber, A. Bräuer, and R. Kowarschik, Phys. Rev. A 66, 063809 (2002).

${ }^{30}$ A. F. Koenderink, M. Kafesaki, C. M. Soukoulis, and V. Sandoghdar, J. Opt. Soc. Am. B 23, 1196 (2006).

${ }^{31}$ S. A. Empedocles, R. Neuhauser, and M. G. Bawendi, Nature (London) 399, 126 (1999).

${ }^{32}$ A. L. Efros, Phys. Rev. B 46, 7448 (1992).
${ }^{33}$ W. L. Barnes, J. Mod. Opt. 45, 661 (1998).

${ }^{34}$ A. F. van Driel, G. Allan, C. Delerue, P. Lodahl, W. L. Vos, and D. Vanmaekelbergh, Phys. Rev. Lett. 95, 236804 (2005).

${ }^{35}$ A. F. van Driel, Ph.D. thesis, University of Utrecht, 2006.

${ }^{36}$ M. Califano, A. Franceschetti, and A. Zunger, Phys. Rev. B 75, 115401 (2007).

${ }^{37}$ X. Fan, M. C. Lonergan, Y. Zhang, and H. Wang, Phys. Rev. B 64, 115310 (2001).

${ }^{38}$ L. M. Ramaniah and S. V. Nair, Phys. Rev. B 47, 7132 (1993).

${ }^{39}$ A. E. Siegman, Lasers (University Science Books, Sausalito, 1986).

${ }^{40}$ Y. Kayanuma, Phys. Rev. B 38, 9797 (1988). 